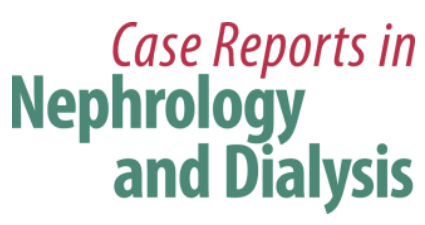

\title{
Antineutrophil Cytoplasmic Antibodies-Negative Pauci-Immune Crescentic Glomerulonephritis Associated with Multiple Myeloma
}

\author{
Cyriacus Uzoma Anaele $^{a} \quad$ Weeraporn Srisung $^{a} \quad$ Yvette Tomacruz $^{b}$ \\ Melvin Laski ${ }^{\mathrm{a}}$ \\ Departments of Internal Medicine, ${ }^{\mathrm{a}}$ Texas Tech University Health Science Center and \\ ${ }^{\mathrm{b}}$ Covenant Medical Center, Lubbock, Tex., USA
}

\section{Key Words}

Antineutrophil cytoplasmic antibodies · Pauci-immune crescentic glomerulonephritis .

Multiple myeloma

\begin{abstract}
Pauci-immune crescentic glomerulonephritis (PICGN) is most commonly associated with antineutrophil cytoplasmic antibodies (ANCA). We report a case of chronic, sclerosing ANCAnegative PICGN discovered when a patient presented with multiple myeloma. A 57-year-old woman presented with complaints of nausea, emesis and weakness. She was found to be in renal failure with a serum creatinine of $9.4 \mathrm{mg} / \mathrm{dl}$, mild hyperkalemia and acidosis. She was noted to have normochromic, normocytic anemia with normal platelet and white cell counts, normal plasma proteins and serum protein electrophoresis. Further studies revealed increased concentrations of $\mathrm{k}$ and $\lambda$ light chains in a ratio of 34.89; a bone marrow biopsy found $12 \%$ plasma cells. Serum protein electrophoresis revealed no spike. ANCA, antiglomerular basement membrane, antineutrophil antibody, hepatitis panel and serum complements were normal. A kidney biopsy result showed chronic sclerosing PICGN plus tubular necrosis, severe tubular atrophy, interstitial fibrosis and severe arteriosclerosis. Congo red stains were negative and electron microscopy showed no intraglomerular deposits. The patient was subsequently treated for myeloma with bortezomib and dexamethasone with good hematologic response but never recovered renal function. She remains on outpatient hemodialysis. Renal manifestations of myeloma often involve glomerular deposition disease, tubulointerstitial disease, with characteristic proteinaceous casts, or both. In contrast, our patient
\end{abstract}




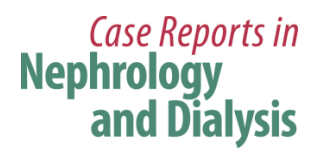

Case Rep Nephrol Dial 2015;5:124-129

DOI: $10.1159 / 000432394$

(C) 2015 S. Karger AG, Basel

www.karger.com/cnd

Anaele et al.: Antineutrophil Cytoplasmic Antibodies-Negative Pauci-Immune Crescentic Glomerulonephritis Associated with Multiple Myeloma

demonstrated neither of these findings but had chronic sclerosing PICGN. Crescentic glomerulonephritis occurring in patients with plasma cell dyscrasias has been previously reported, but the association remains extremely rare.

(c) 2015 S. Karger AG, Basel

\section{Introduction}

Multiple myeloma accounts for $1 \%$ of all malignancies and approximately $10 \%$ of hematologic malignancies $[1,2]$. Approximately $70 \%$ of patients with multiple myeloma have urinary $M$ protein spike and about $20-25 \%$ have kidney disease at the time of diagnosis. Up to half of myeloma patients demonstrate renal involvement during the course of their disease $[3,4]$. Cast nephropathy or 'myeloma kidney' is implicated in about $41 \%$ of cases and is distinctive for multiple myeloma [5]. Monoclonal immunoglobulin deposition disease, primary amyloidosis, proximal and distal tubulopathy, renal vein thrombosis, type 1 cryoglobulinemia, proliferative glomerulonephritis, interstitial nephritis, plasma cell infiltration, urate deposition disease, pyelonephritis and nephrocalcinosis represent less common forms of renal involvement [6-9]. Rapidly progressive glomerulonephritis in multiple myeloma is rare; cases reported thus far typically demonstrate proliferative glomerulonephritis and/or glomerular deposits on renal biopsy. We here report a case of antineutrophil cytoplasmic antibodies (ANCA)-negative, pauci-immune, chronic sclerosing, crescentic glomerulonephritis without deposits on electron microscopy, which was discovered at the time the patient presented with multiple myeloma and which resulted in end-stage kidney disease.

\section{Case Report}

A 57-year-old Hispanic woman was admitted with nausea, vomiting and weakness of 1 week duration. She described herself to be in good health but had not seen a doctor for 15 years. Her history was only significant for pregnancies and visits to the emergency room for epistaxis 3 years prior to admission and after a fall 6 years prior to admission. She used no routine home medications and she had no known drug allergies. She has 3 children in good health. She denied smoking and the use of alcohol or illicit drugs. Her father died from malignancy, type unknown. Physical examination on admission was normal except for pallor. Initial laboratory findings are presented in table 1.

The patient was started on hemodialysis on admission and was transfused with 2 units of packed red blood cells for severe anemia. Urinalysis was positive for microscopic hematuria, and the patient had positive urine $M$ spike with faint $\kappa$ light chain on serum immunofixation (table 1). Renal biopsy on day 4 of admission was consistent with chronic sclerosing pauci-immune glomerulonephritis (fig. 1). Neither immunofluorescence nor electron microscopy showed evidence of an immune-mediated glomerulonephritis. 'Burnt-out' pauciimmune crescentic glomerulonephritis was therefore diagnosed.

Further investigations to evaluate myeloma showed a negative bone survey and bone scan. Initial bone marrow biopsy showed monoclonal plasmacytosis (12\%) consistent with plasma cell myeloma. There was no evidence of increased blasts, monoclonal B-cell or aberrant T-cell process. The patient was eventually discharged home with hematology/oncology follow-up and remains on outpatient hemodialysis. She was started on high-dose dexamethasone a few days after discharge and bortezomib $1.3 \mathrm{mg} / \mathrm{m}^{2}$ twice weekly after hemodialysis approximately 1 month after discharge. 


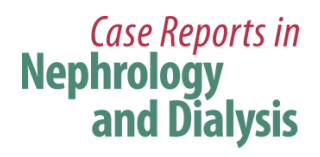

Case Rep Nephrol Dial 2015;5:124-129

DOI: $10.1159 / 000432394$

c) 2015 S. Karger AG, Basel

www.karger.com/cnd

Anaele et al.: Antineutrophil Cytoplasmic Antibodies-Negative Pauci-Immune Crescentic Glomerulonephritis Associated with Multiple Myeloma

She was readmitted 3 months later with fever and symptoms of upper respiratory tract infection and was treated with antibiotics. The immunological investigations were repeated because of the rarity of multiple myeloma presenting with pauci-immune crescentic glomerulonephritis. These studies again demonstrated low IgG, IgA and IgM. However, repeat serum protein electrophoresis showed no $\mathrm{M}$ spike, and serum immunofixation showed a polyclonal pattern. Repeat free $\kappa / \lambda$ ratio was now 10.25 . Bone marrow aspirate Congo red stain was negative for amyloid deposits. C-ANCA and P-ANCA were again both negative. Spot urine protein was $834 \mathrm{mg} / \mathrm{dl}$, the urine creatinine concentration was $24 \mathrm{mg} / \mathrm{dl}$, the urine albumin concentration was $35 \mathrm{mg} / \mathrm{dl}$ and the urine protein/creatinine ratio was 35 . The urine protein electrophoretic pattern was consistent with mixed glomerular and tubular proteinuria. A repeat bone survey showed mild generalized osteopenia, but it revealed no focal lesions. A follow-up bone marrow biopsy after 4 months of bortezomib and dexamethasone therapy showed a normocellular marrow without increased plasma cells. She continues to require hemodialysis despite reduction of the free light chain burden and disappearance of monoclonal plasma cells in the bone marrow.

\section{Discussion}

Crescentic glomerulopathy is a rare complication of multiple myeloma first described by Kaplan and Kaplan [10] in 1970 in a 49-year-old patient with renal failure due to IgG $\lambda$ myeloma. Three cases of extracapillary proliferative glomerulonephritis were reported by Meyrier et al. [11], of which two were due to plasma cell dyscrasias and one was due to Waldenstrom's macroglobulinemia. Lapenas et al. [12] described a patient with diabetes mellitus who developed rapid progressive renal failure over the course of 5 weeks that led to chronic hemodialysis caused by $\kappa$ light chain myeloma.

Grundmann et al. [13] reported a 60-year-old male patient who had a relapse of rapidly progressive glomerulonephritis 10 months after an initial episode that responded to cyclophosphamide and steroid therapy. This time, he had an extensive investigation that revealed $\kappa$ light chain monoclonal gammopathy treated with 2 injections of rituximab followed by 2 cycles of bortezomib therapy resulting in clinical remission.

Our patient presented with advanced renal disease, hence renal recovery was unlikely. Perhaps, if she had presented early, she may have recovered her renal function with bortezomib and dexamethasone treatment. The exact mechanism of renal injury in this case remains unclear and needs further investigation since there were no demonstrable immune complexes, monoclonal deposition disease or cast nephropathy. One possible theory could be a form of direct toxic injury to the glomerular basement membrane by the free light chains with subsequent disruption of the basement membrane and formation of fibrocellular crescents. Whether this is preceded by extracapillary and/or endocapillary proliferation remains unclear. There could also be concurrent direct toxic injury to tubular cells by $\kappa$ light chains and interstitial nephritis.

\section{Conclusion}

Multiple myeloma associated with sclerosing pauci-immune crescentic glomerulonephritis is rare. This case highlights an atypical renal pathologic manifestation of multiple myeloma and underscores that multiple myeloma should be considered in the differential diagnosis of rapidly progressive glomerulonephritis, especially if there is heavy proteinuria. 
Anaele et al:: Antineutrophil Cytoplasmic Antibodies-Negative Pauci-Immune Crescentic Glomerulonephritis Associated with Multiple Myeloma

In such patients, early screening for serum free light chains followed by renal biopsy and bone marrow biopsy can establish the diagnosis and guide therapy.

\section{Disclosure Statement}

The authors declare that no financial or other conflict of interest exists in relation to the content of the article.

\section{References}

1 Kyle RA, Therneau TM, Rajkumar SV, et al: Incidence of multiple myeloma in Olmsted County, Minnesota: trend over 6 decades. Cancer 2004;101:2667-2674.

-2 Smith A, Howell D, Patmore R, et al: Incidence of haematological malignancy by sub-type: a report from the Haematological Malignancy Research Network. Br J Cancer 2011;105:1684-1692.

-3 Knudsen LM, Hippe E, Hjorth M, Holmberg E, Westin J: Renal function in newly diagnosed multiple myeloma - a demographic study of 1,353 patients. The Nordic Myeloma Study Group. Eur J Haematol 1994;53:207212.

-4 Kyle RA, Gertz MA, Witzig TE, Lust JA, Lacy MQ, Dispenzieri A, Fonseca R, Rajkumar SV, Offord JR, Larson DR, Plevak ME, Therneau TM, Greipp PR: Review of 1,027 patients with newly diagnosed multiple myeloma. Mayo Clin Proc 2003;78:21-33.

5 Montseny JJ, Kleinknecht D, Meyrrier A, et al: Long-term outcome according to renal histological lesions in 118 patients with monoclonal gammopathies. Nephrol Dila Transplant 1998;13:1438-1445.

6 Crosthwaite A, Mount P: Rapidly progressive glomerulonephritis complicating primary AL amyloidosis and multiple myeloma. Nephrol Dial Transplant 2010;25:2786-2789.

7 Hill GS, Morel-Maroger L, Méry JP, et al: Renal lesions in multiple myeloma: their relationship to associated protein abnormalities. Am J Kidney Dis 1983;2:423-438.

8 Iványi B: Renal complications in multiple myeloma. Acta Morphol Hung 1989;37:235-243.

-9 Lorenz EC, Sethi S, Poshusta TL, et al: Renal failure due to combined cast nephropathy, amyloidosis and light-chain deposition disease. Nephrol Dial Transplant 2010;25:1340-1343.

10 Kaplan NG, Kaplan KC: Monoclonal gammopathy, glomerulonephritis and the nephrotic syndrome. Arch Intern Med 1970;125:696-700.

11 Meyrier A, Simon P, Mignon F, Striker L, Ramèe M: Rapidly progressive ('crescent') glomerulonephritis and monoclonal gammopathies. Nephron 1984;38:156-162.

12 Lapenas DJ, Drewry SJ, Luke RL III, et al: Crescentic light-chain glomerulopathy. Report of a case. Arch Pathol Lab Med 1983;107:319-323.

-13 Grundmann G, Witthus M, Gobel H, Kisner T, Siewert R, Benzing T, Kurschat C: Monoclonal gammopathyassociated pauci-immune extracapillary-proliferative glomerulonephritis successfully treated with bortezomib. Clin Kidney J 2013;6:327-329. 
Anaele et al.: Antineutrophil Cytoplasmic Antibodies-Negative Pauci-Immune Crescentic Glomerulonephritis Associated with Multiple Myeloma

Table 1. Results of initial laboratory investigations

\begin{tabular}{|c|c|c|c|}
\hline $\begin{array}{l}\text { Serum chemistry } \\
\mathrm{Na}: 141 \mathrm{mEq} / \mathrm{l} \\
(134-145 \mathrm{mEq} / \mathrm{l})\end{array}$ & $\begin{array}{l}\mathrm{K}: 5.2 \mathrm{mEq} / \mathrm{l} \\
(3.5-5.3 \mathrm{mEq} / \mathrm{l})\end{array}$ & $\begin{array}{l}\text { Creatinine: } 9.4 \mathrm{mg} / \mathrm{dl} \\
(0.7-1.2 \mathrm{mg} / \mathrm{dl})\end{array}$ & $\begin{array}{l}\text { Glucose: } 95 \mathrm{mg} / \mathrm{dl} \\
(65-110 \mathrm{mg} / \mathrm{dl})\end{array}$ \\
\hline $\begin{array}{l}\mathrm{Cl}: 110 \mathrm{mEq} / \mathrm{l} \\
(98-108 \mathrm{mEq} / \mathrm{l})\end{array}$ & $\begin{array}{l}\mathrm{HCO}_{3}: 19 \mathrm{mEq} / \mathrm{l} \\
(22-31 \mathrm{mEq} / \mathrm{l})\end{array}$ & $\begin{array}{l}\text { BUN: } 72 \mathrm{mg} / \mathrm{dl} \\
(7-18 \mathrm{mg} / \mathrm{dl})\end{array}$ & \\
\hline $\begin{array}{l}\text { Mg: } 1.9 \mathrm{mg} / \mathrm{dl} \\
(1.8-2.2 \mathrm{mg} / \mathrm{dl})\end{array}$ & $\begin{array}{l}\text { Ca: } 8.4 \mathrm{mg} / \mathrm{dl} \\
(8.4-11 \mathrm{mg} / \mathrm{dl})\end{array}$ & $\begin{array}{l}\text { Albumin: } 3.3 \mathrm{~g} / \mathrm{dl} \\
(3.4-5.0 \mathrm{~g} / \mathrm{dl})\end{array}$ & $\begin{array}{l}\text { Total protein: } 6.5 \mathrm{~g} / \mathrm{dl} \\
(5.5-7.8 \mathrm{~g} / \mathrm{dl})\end{array}$ \\
\hline $\begin{array}{l}\text { AST: } 10 \mathrm{U} / \mathrm{l} \\
(5-45 \mathrm{U} / \mathrm{l})\end{array}$ & $\begin{array}{l}\text { ALT: }<8 \mathrm{U} / \mathrm{l} \\
(<8 \mathrm{U} / \mathrm{l})\end{array}$ & $\begin{array}{l}\text { Alkaline phosphatase: } 66 \mathrm{U} / \mathrm{l} \\
(38-126 \mathrm{U} / \mathrm{l})\end{array}$ & \\
\hline $\begin{array}{l}\text { Complete blood count } \\
\text { Hemoglobin: } 7.3 \mathrm{~g} / \mathrm{dl} \\
(12-16 \mathrm{~g} / \mathrm{dl})\end{array}$ & $\begin{array}{l}\text { Hematocrit: } 20.4 \% \\
(37-47 \%)\end{array}$ & $\begin{array}{l}\text { WBC: } 8,300 / \mathrm{mm}^{3} \\
\left(3,600-10,800 / \mathrm{mm}^{3}\right)\end{array}$ & $\begin{array}{l}\text { Platelets: } 153,000 / \mathrm{mm}^{3} \\
\left(150,000-400,000 / \mathrm{mm}^{3}\right)\end{array}$ \\
\hline $\begin{array}{l}\text { Iron studies } \\
\text { Iron: } 38 \mu \mathrm{g} / \mathrm{dl} \\
(30-160 \mu \mathrm{g} / \mathrm{dl}) \\
\end{array}$ & $\begin{array}{l}\text { TIBC: } 299 \mu \mathrm{g} / \mathrm{dl} \\
(185-515 \mu \mathrm{g} / \mathrm{dl})\end{array}$ & $\begin{array}{l}\text { Ferritin: } 62 \mathrm{ng} / \mathrm{ml} \\
(13-150 \mathrm{ng} / \mathrm{ml})\end{array}$ & $\begin{array}{l}\text { Transferrin: } 212 \mathrm{mg} / \mathrm{dl} \\
(200-360 \mathrm{mg} / \mathrm{dl})\end{array}$ \\
\hline $\begin{array}{l}\text { Viral hepatitis serology } \\
\text { HCV Ab: nonreactive }\end{array}$ & HBS Ag: nonreactive & HepB core Ab: nonreactive & HBS Ab, quant.: $0.6 \mathrm{mIU} / \mathrm{ml}$ \\
\hline $\begin{array}{l}\text { Immunology investigations } \\
\text { Antiproteinase } 3 \mathrm{Ab} \\
\text { (C-ANCA): negative }\end{array}$ & $\begin{array}{l}\text { ANA titer: }<100 \mathrm{AU} / \mathrm{ml} \\
(0-100 \mathrm{AU} / \mathrm{ml})\end{array}$ & $\begin{array}{l}\text { Antihistone } \mathrm{Ab}: 4 \mathrm{AU} / \mathrm{ml} \\
(0-100 \mathrm{AU} / \mathrm{ml})\end{array}$ & $\begin{array}{l}\text { Scl-70 Ab: } 2 \mathrm{AU} / \mathrm{ml} \\
(0-100 \mathrm{AU} / \mathrm{ml})\end{array}$ \\
\hline $\begin{array}{l}\text { Myeloperoxidase } \mathrm{Ab} \\
\text { (p-ANCA): negative }\end{array}$ & $\begin{array}{l}\text { SSA Ab: } 6 \mathrm{AU} / \mathrm{ml} \\
(0-100 \mathrm{AU} / \mathrm{ml})\end{array}$ & $\begin{array}{l}\text { RNP Ab: } 6 \mathrm{AU} / \mathrm{ml} \\
(0-100 \mathrm{AU} / \mathrm{ml})\end{array}$ & $\begin{array}{l}\text { Jo } 1 \mathrm{Ab}: 4 \mathrm{AU} / \mathrm{ml} \\
(0-100 \mathrm{AU} / \mathrm{ml})\end{array}$ \\
\hline $\begin{array}{l}\text { Anti-glomerular basement } \\
\text { membrane } \mathrm{Ab} \text { : negative }\end{array}$ & $\begin{array}{l}\text { Smith Ab: } 4 \mathrm{AU} / \mathrm{ml} \\
(0-100 \mathrm{AU} / \mathrm{ml})\end{array}$ & $\begin{array}{l}\text { Anti-double strand DNA Ab: } \\
7 \mathrm{IU} / \mathrm{ml}(0-100 \mathrm{IU} / \mathrm{ml})\end{array}$ & $\begin{array}{l}\text { Rheumatoid factor: } 8 \mathrm{IU} / \mathrm{l} \\
(0-14 \mathrm{IU} / \mathrm{l})\end{array}$ \\
\hline $\begin{array}{l}\text { Serum electrophoresis } \\
\alpha 1 \text { globulin: } \\
0.35 \mathrm{~g} / \mathrm{dl}\end{array}$ & $\begin{array}{l}\alpha 2 \text { globulin: } \\
0.89 \mathrm{~g} / \mathrm{dl}\end{array}$ & $\begin{array}{l}\beta \text { globulins: } \\
1.06 \mathrm{~g} / \mathrm{dl}\end{array}$ & $\begin{array}{l}\gamma \text { globulins: } \\
0.92 \mathrm{~g} / \mathrm{dl}\end{array}$ \\
\hline $\begin{array}{l}\text { Urine electrophoresis } \\
\text { Total protein: } 137\end{array}$ & Albumin: 55.78 (40.72\%) & $\gamma$ globulin: $40.68(29.69 \%)$ & M spike: 21.12 \\
\hline $\begin{array}{l}\text { Serum free light chains } \\
\kappa: 1,827 \mathrm{mg} / \mathrm{l} \\
(3.3-19.5 \mathrm{mg} / \mathrm{l})\end{array}$ & $\begin{array}{l}\lambda: 52.4 \mathrm{mg} / \mathrm{l} \\
(5.7-26.5 \mathrm{mg} / \mathrm{l})\end{array}$ & $\kappa / \lambda$ ratio: 34.9 & $\begin{array}{l}\text { Serum immunofixation shows faint } \\
\kappa \text { light chain }\end{array}$ \\
\hline $\begin{array}{l}\text { Urinalysis } \\
\text { Color: yellow }\end{array}$ & $\begin{array}{l}\text { Urine clarity: cloudy } \\
\text { (clear) }\end{array}$ & $\begin{array}{l}\text { Specific gravity: } 1.015 \\
(1.005-1.030)\end{array}$ & $\begin{array}{l}\text { Urine } \mathrm{pH}: 7.0 \\
(5.0-7.5)\end{array}$ \\
\hline $\begin{array}{l}\text { Urine protein: } 100 \mathrm{mg} / \mathrm{dl} \\
\text { (negative) }\end{array}$ & $\begin{array}{l}\text { Glucose: negative } \\
\text { (negative, } \mathrm{mg} / \mathrm{dl} \text { ) }\end{array}$ & $\begin{array}{l}\text { Ketones: negative } \\
\text { (negative, } \mathrm{mg} / \mathrm{dl} \text { ) }\end{array}$ & $\begin{array}{l}\text { Bilirubin: negative } \\
\text { (negative) }\end{array}$ \\
\hline $\begin{array}{l}\text { Urine blood: large } \\
\text { (negative) }\end{array}$ & $\begin{array}{l}\text { Urine nitrite: negative } \\
\text { (negative) }\end{array}$ & $\begin{array}{l}\text { Leukocyte esterase: moderate } \\
\text { (negative) }\end{array}$ & $\begin{array}{l}\text { Urine RBCs: } 30-40 / \mathrm{hpf} \\
(0-3 / \mathrm{hpf})\end{array}$ \\
\hline $\begin{array}{l}\text { Urine WBCs: } 20-30 / \mathrm{hpf} \\
(0-5 / \mathrm{hpf})\end{array}$ & $\begin{array}{l}\text { Squamous epithelial cells: } \\
3-5 / \mathrm{hpf}(0-5 / \mathrm{hpf})\end{array}$ & $\begin{array}{l}\text { Bacteria: light } / \mathrm{hpf} \\
\text { (negative/hpf) }\end{array}$ & \\
\hline
\end{tabular}

Normal values are presented in parentheses. BUN = Blood urea nitrogen; AST = aspartate transaminase; ALT = alanine transaminase; $\mathrm{WBC}=$ white blood cell; $\mathrm{TIBC}=$ total iron-binding capacity; $\mathrm{HCV}=$ hepatitis $\mathrm{C}$ virus; $\mathrm{Ab}=$ antibody; HBS = hepatitis B surface; $\mathrm{Ag}=$ antigen; $\mathrm{HepB}=$ hepatitis $\mathrm{B} ;$ quant. = quantitative; ANA = antineutrophil antibody; SSA = Sjörgen's syndromerelated antigen $\mathrm{A} ; \mathrm{RNP}=$ ribonucleoprotein; $\mathrm{RBCs}=$ red blood cells; $\mathrm{hpf}=$ high-power field. 


\section{Case Reports in \\ Nephrology and Dialysis}

\begin{tabular}{l|l}
\hline \multicolumn{2}{l}{ Case Rep Nephrol Dial 2015;5:124-129 } \\
\hline DOI: 10.1159/000432394 & $\begin{array}{l}\text { ○ 2015 S. Karger AG, Basel } \\
\text { www.karger.com/cnd }\end{array}$ \\
\hline
\end{tabular}

Anaele et al:: Antineutrophil Cytoplasmic Antibodies-Negative Pauci-Immune Crescentic Glomerulonephritis Associated with Multiple Myeloma
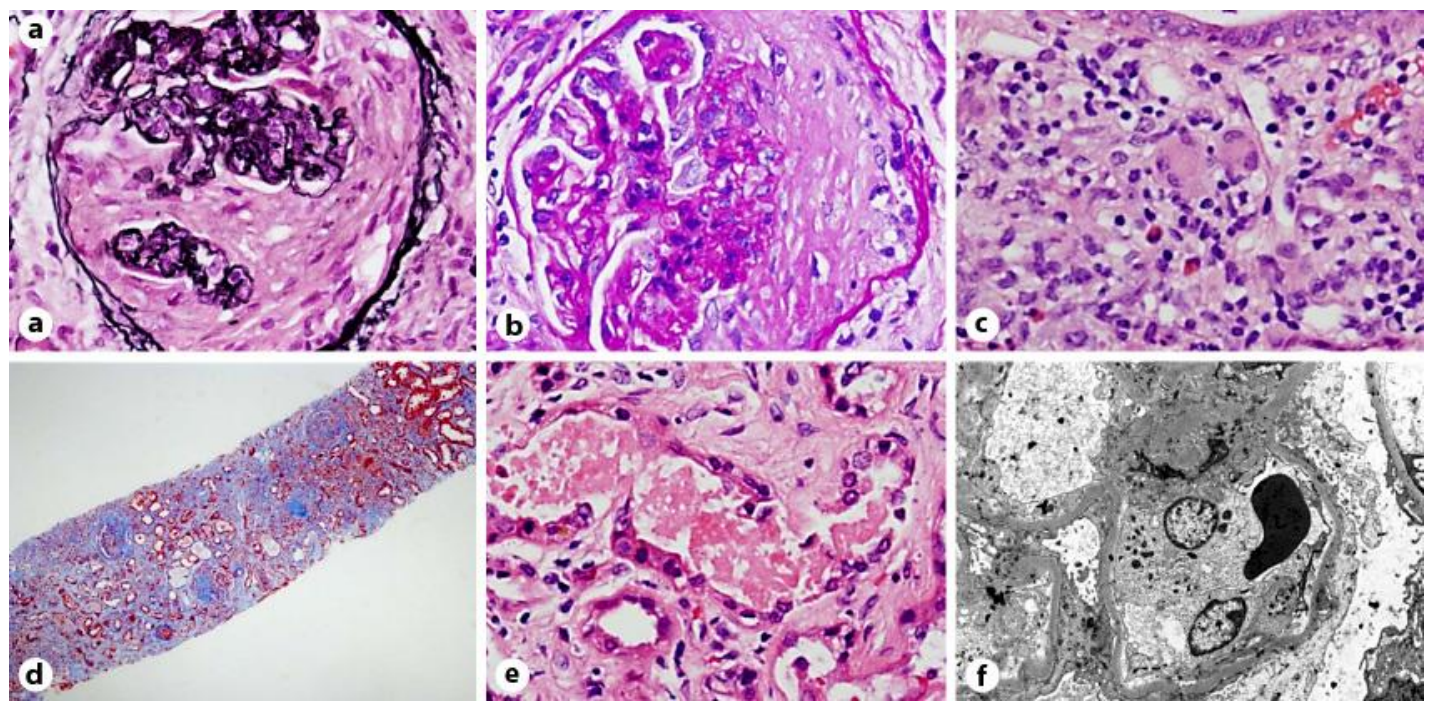

Fig. 1. Renal biopsy images. a-e Light microscopy shows fragments of renal cortex and medulla with up to 7 glomeruli present, 1 of which is globally sclerotic. Three glomeruli have an increased mesangial matrix, but no mesangial hypercellularity was seen. The capillary walls are segmentally thickened, but no 'spikes' and 'holes' are seen with silver stain. No active cellular crescents, fibrinoid necrosis or intracapillary hypercellularity is seen. There is a severe acute tubular injury consisting of epithelial cell necrosis, apical blebbing, epithelial cell sloughing, tubular dilatation and reparative changes. No red cell casts are seen. There is a moderate to severe interstitial infiltrate consisting of lymphocytes, plasma cells, scattered eosinophils and rare granulomas. Tubular atrophy and interstitial fibrosis is seen in $80 \%$ of the cortical sample. The larger arteries have severe fibrointimal thickening and reduplication of the internal elastic lamina. The arterioles have mild hyalinosis but no vasculitis or fibrinoid necrosis of the vessel walls. f Electron microscopy. The specimen consists of minute fragments of renal cortex and medulla with up to 4 glomeruli present; 1 is globally sclerotic, 1 glomerulus has a fibrocellular crescent, and 1 glomerulus has a fibrous crescent. The glomerular basement membrane has normal thickness; no subepithelial, subendothelial or intramembranous electron dense deposits are seen. There is a mild increase in mesangial matrix, but no mesangial deposits are seen. No tubuloreticular inclusions are noted. There is moderate podocyte foot process effacement involving approximately $60 \%$ of the glomerular basement membrane surface. 